\title{
Flora das cangas da Serra dos Carajás, Pará, Brasil: Portulacaceae
}

\author{
Flora of the canga of Serra dos Carajás, Pará, Brazil: Portulacaceae
}

Ana Maria Giulietti ${ }^{1,3} \&$ Alexa Paes Coelho ${ }^{2}$

\begin{abstract}
Resumo
É apresentado o tratamento florístico das espécies de Portulacaceae que ocorrem sobre as cangas da Serra dos Carajás, no estado do Pará, Brasil. Um gênero e duas espécies foram registradas: Portulaca oleracea e $P$. sedoides. São incluídas chaves, descrições, ilustrações, fotografias em campo e comentários sobre as espécies. Palavras-chave: Amazônia, Caryophyllales, Floresta Nacional de Carajás, florística, taxonomia.
\end{abstract}

\begin{abstract}
We present the floristic treatment for the species of Portulacaceae from the canga of the Serra dos Carajás, state of Pará, Brazil. One genus and two species were recorded: Portulaca oleracea and P. sedoides. Keys, descriptions, illustrations, photos, and comments are also provided.

Key words: Amazonia, Caryophyllales, Carajás National Forest, floristic, taxonomy.
\end{abstract}

\section{Portulacaceae}

A delimitação de Portulacaceae Juss. tem suscitado discussão especialmente na delimitação da família em relação às outras de Caryophyllales. Segundo Nyffeler \& Eggli (2010), Ocampo \& Columbus (2012) e Hernández-Ledesme et al. (2015), Portulacaceae forma uma linhagem de plantas suculentas com Cactaceae, Anacampserotaceae e Talinaceae. Cactaceae distingue-se das outras famílias pela presença de aréolas que portam espinhos e folhas (nas espécies que essas ocorrem) e fruto baga carnosa. Anacampserotaceae e Talinaceae foram desmembradas de Portulacaceae, e compartilham a ausência de espinhos e os frutos secos e deiscentes. Portulacaceae distingue-se de ambas por apresentar o fruto com deiscência transversal (pixídio) e não em valvas, e inflorescência terminal cimosa, muito congesta e capituliforme.

Portulacaceae é reconhecida na APG IV (2016), como uma família monotípica incluindo apenas Portulaca L. e cerca de 110 espécies. Os outros 26 gêneros incluídos anteriormente na família por Carolin (1993), foram desemembrados para compor as novas famílias como Montiaceae Raf. (13 gêneros), Anacampserotaceae Eggli \& Nyffeler (três gêneros) e Talinaceae Doweld (três gêneros). Dos gêneros restantes aceitos em Carolin (1993), cinco foram sinonimizados em Montia L. (Montiaceae) e três foram incorporados em Didiereaceae Radlk. O gênero Talinum Adans como delimitado em Carolin (1993) teve as 30 espécies de folhas cilíndricas segregado no gênero Phemeranthus Raf. e incluído em Montiaceae, enquanto Talinum s.s. com cerca de 15 espécies e folhas planas, foi incluído em Talinaceae (Carolin 1993; Hernández-Ledesme et al. 2015).

No Brasil, além de Portulacaceae ocorre apenas Talinaceae, com duas espécies de Talinum (BFG 2015), que não ocorrem na Serra dos Carajás. Portulacaceae com o único gênero Portulaca tem distribuição cosmopolita, mas concentrada especialmente no Hemisfério Sul.

\section{Portulaca L. \\ O gênero Portulaca é caracterizado por incluir ervas suculentas, semi-prostradas, prostradas ou eretas, com caules simples ou ramificados desde a base, com nós que portam tricomas axilares, brancos a castanhos com diversos tamanhos e densidades ou raramente escamas. As folhas são alternas, sub-opostas ou opostas, com lâminas achatadas, elípticas ou cilíndricas em corte transversal, geralmente glabras, e margens}

\footnotetext{
${ }^{1}$ Instituto Tecnológico Vale, R. Boaventura da Silva 955, Nazaré, 66055-090, Belém, PA, Brasil.

${ }^{2}$ Universidade do Estado da Bahia - UNEB, Rod. Alagoinhas/Salvador, BR-110, Km 03, 48040-210, Alagoinhas, BA, Brasil.

${ }^{3}$ Autor para correspondência: ana.giulietti@itv.org
} 
inteiras. As inflorescências são terminais, cimosas, capituliformes com invólucro de folhas, geralmente ligeiramente diferenciadas das vegetativas, ou raramente, flores isoladas. As flores são sésseis a curto pediceladas; actinomorfas; bissexuadas; com 2 sépalas desiguais; 4-5 pétalas membranáceas; 4-muitos estames; ovário ínfero, estilete 1, com 2-12 ramos estigmáticos. $\mathrm{O}$ fruto é do tipo pixídio e as sementes são lisas ou com diferentes padrões de escultura (Carolin 1993; Ocampo \& Columbus 2012; Ocampo 2013).

Apesar da circunscrição de Portulaca ser aceita por todos os especialistas de Caryophyllales, o número de espécies nele incluídas e as propostas infra-genéricas ainda estão em discussão. O número de espécies varia de 40 (Geesink 1969) até pouco mais de 100 (Legrand 1958; Carolin 1993) ou 200 (Willis 1966). A variação no número de espécies está associada principalmente a grande diversidade morfológica apresentada pelas diferentes populações que compõem cada espécie, especialmente aquelas de ampla distribuição, como por exemplo as pantropicais $P$. oleracea L. e $P$. quadrifida L. e as neotropicais $P$. halimoides L. e P. pilosa L. Caracteres da semente, especialmente a escultura, têm sido considerados como muito importantes na distinção das espécies. Ocampo (2013) estudou 49 espécies, e propôs nova nomenclatura que foi utilizada para descrever a escultura das sementes nesse trabalho. Concluiu que para várias espécies, a escultura da semente permite a sua identificação correta, mas outras, especialmente as de grande distribuição geralmente apresentam variações entre as populações, dificultando a correta identificação.

A divisão infragenérica de Portulaca proposta por Legrand $(1958,1962)$ é questionada com base em análises filogenéticas (Ocampo \& Columbus 2012). Para esses autores, o gênero é monofilético e tem duas linhagens principais: as plantas com folhas opostas, distribuídas na África, Ásia e Austrália e, as plantas com folhas alternas ou subopostas, das Américas.

Nas cangas da Serra dos Carajás, Portulacaceae está representada por Portulaca oleracea e $P$. sedifolia.

\section{Chave de identificação das espécies de Portulaca ocorrentes nas cangas da Serra dos Carajás}

1. Limbo foliar 6-18 × 4-7 mm, oboval a espatulado, cálice com sépalas carenadas no dorso, corola amarela, ramos estigmáticos 5 1.1. Portulaca oleracea

1'. Limbo foliar 4-7 × 1,5-2,5 mm, linear a elíptico-lanceolado, cálice com sépalas não carenadas, corola branca a rosada, ramos estigmáticos 3 1.2. Portulaca sedifolia

\subsection{Portulaca oleracea L. Sp. Pl. 445. 1753.}

Fig. 1a-c

Erva anual, suculenta, caule com ramificação dicotômica, raramente não dicotômica na porção apical, ramos prostrados, 7,5-10 cm compr., verdes a avermelhados, tricomas axilares finos, brancos, 1-2 mm compr. Folhas opostas a subopostas, sésseis ou pecíolos até $2 \mathrm{~mm}$ compr.; limbo 6-18 $\times$ 4-7 mm, oboval a espatulado, achatado em corte transversal, em material fresco, nervura central evidente na face abaxial; base arredondada; ápice arredondado; margem inteira com pequena borda avermelhada, em material fresco; glabras, geralmente persistentes. Folhas involucrais 3-5 por inflorescência, sem distinção de tamanho e forma das folhas dos ramos. Inflorescência 2-6-flora, com uma flor abrindo de cada vez. Flores sésseis; sépalas ca. $3 \mathrm{~mm}$ compr., glabras, dorso carenado, ápice agudo; pétalas 5, amarelas, obcordadas ca. $2 \times 1,5 \mathrm{~mm}$, ápice arredondado; estames 6-12, filetes ca. 1,5 mm; estilete ca. 1,5 mm compr.; ramos estigmáticos 5, ca. 1,5 mm compr. Pixídio ca. $3 \mathrm{~mm}$ compr., séssil, porção basal ca. 1,5 $\mathrm{mm}$, opérculo ca. $2 \mathrm{~mm}$, cônico, ápice obtuso, abertura na região mediana, linha de deiscência não espessada. Sementes subreniformes, castanhoescuras a negras, ca. 20 por fruto, opacas, 0,4-0,5 $\mathrm{mm}$, faces laterais e dorsal com células individuais alongadas, paredes periclinais pouco convexas, com projeções acessórias convexas e diminutas.

Material examinado: Canaã dos Carajás, FLONA de Carajás, no caminho para Mina do Sossego, afloramento de granito, 6 ${ }^{\circ} 17 ' 52$ ' $\mathrm{S}, 50^{\circ} 05^{\prime} 22^{\prime}$ 'W, 15.II.2017, D.C. Zappi et al. 3521 (MG).

Portulaca oleracea é de fácil reconhecimento entre as espécies do Brasil, tanto em campo como em herbário, por apresentar as sépalas carenadas na parte dorsal, evidentes no botão floral, associadas às folhas opostas pelo menos na porção apical do ramo. Linnaeus (1753) ao descrever a espécie colocou sob o mesmo nome, tanto as formas cultivadas, como as naturais. As formas cultivadas, 
conhecidas com o nome de "purslane"- são formadas por plantas muito maiores e robustas, e devido às folhas suculentas e ligeiramente salgadas, são utilizadas na alimentação (saladas, sopas) nos últimos 4.000 anos. Essas plantas foram descritas como Portulaca sativa Haw. e posteriormente o táxon foi considerado como uma das variedades de $P$. oleracea. Uotila et al. (2012) consideraram $P$. oleracea e $P$. sativa como espécies distintas sendo essa última de ocorrência em várias áreas de países da Europa (Finlândia, Suiça, Alemanha, Italia, Bélgica), da Ásia (Israel) ou da Europa/Ásia (Russia e Turkia). Tal conceito levou a redução da área de distribuição de $P$. oleracea, que era considerada como quase cosmopolita e atualmente tem distribuição pantropical. Nas Américas, $P$. oleracea ocorre dos sul dos Estados Unidos até a Argentina. No Brasil, onde recebe muito nomes populares como Beldroega, Bredo-de-porco, Capanga, Ora-pro-nobis, Porcelana, Salada-de-

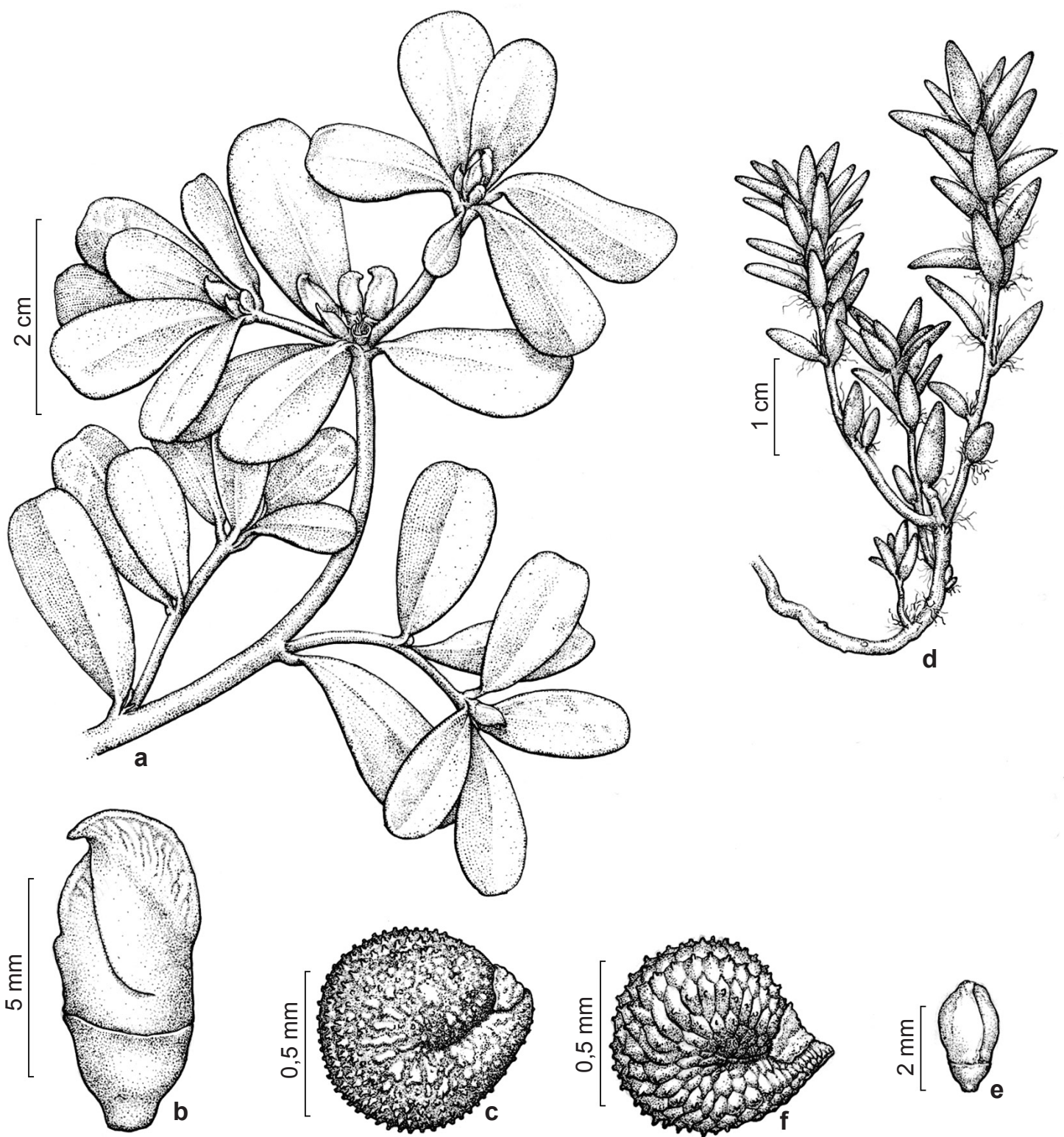

Figura 1 -a-c. Portulaca oleracea - a. hábito; b. botão floral fechado; c. semente. d-f. Portulaca sedifolia - d. hábito; e. botão floral fechado; f. semente. (a-c. D.C. Zappi 3521; d-f. R. Harley 57495)

Figure 1 - a-c. Portulaca oleracea - a. habit; b. closed flower bud; c. seed. d-f. Portulaca sedifolia - d. habit; e. closed flower bud; f. seed. (a-c. D.C. Zappi 3521; d-f. R. Harley 57495) 
negro, ocorre em todos os estados, sempre associada com vegetação aberta, mas principalmente em ambientes ruderais (Coelho 2006; Coelho \& Giulietti 2010; BFG 2015).

$\mathrm{Na}$ Serra dos Carajás tem apenas um registro em herbário, associado a afloramento de granito. Porém, é possível que ocorra esporadicamente em outras áreas, pois é considerada como “espécie daninha" (Lorenzi 2000).

1.2. Portulaca sedifolia N.E.Br, Trans. Linn. Soc. London, Bot., ser. 2,6: 19. 1901.

Figs. 1d-f; 2a-h

Erva anual, suculenta, caule com ramificação não dicotômica ou com ramificação dicotômica na porção apical, ramos semi-prostrados a prostrados, 3-10(-22) cm compr., geralmente vermelhovináceos, tricomas axilares muito finos, delicados, brancos, 3-4 mm compr. Folhas alternas, pecíolo ca. 0,5 mm compr.; limbo 4-7 × 1,5-2,5 mm, linear a elíptico-lanceolado, elíptico em seção transversal, em material fresco, nervura central não evidente; base arredondada a obtusa; ápice obtuso a subagudo; margem inteira sem borda; glabras, caducas durante a senescência. Folhas involucrais 6-8 por inflorescência, mais ou menos verticiladas em receptáculo obcônico, pecíolo 0,5-1 mm compr., limbo 4,5-5,5 × 0,8-1 mm, linear; base arredondada; ápice obtuso a subagudo, margem inteira; glabras. Inflorescência 2-3-flora, com uma flor abrindo de cada vez. Flores sésseis; sépalas ca. 2 mm compr., glabras, dorso côncavo, não carenado, ápice agudo; pétalas 5, brancas a rosadas, obcordadas ca. $4 \times 3 \mathrm{~mm}$, ápice emarginado ou arredondado; estames 10, filetes ca. $2 \mathrm{~mm}$ compr.; estilete ca. $2 \mathrm{~mm}$ compr., ramos estigmáticos 3 , ca. $1,5 \mathrm{~mm}$ compr., estigmas alongados ca. 0,8 mm compr. Pixídio 3,2-3,5 mm compr., séssil ou pedicelo até 0,2 mm, porção basal $0,8-1 \mathrm{~mm}$, opérculo 2-2,3 $\mathrm{mm}$, cônico, ápice umbonado, abertura bem abaixo do meio, linha de deiscência espessada. Sementes subreniformes, castanho-escuras a negras, 5-16 por fruto, opacas, 0,6-0,7 mm compr., faces laterais com células individuais alongadas a isodiamétricas, a isodiamétricas, paredes anticlinais irregularmente curvadas a retas, paredes periclinais convexas com diminutas projeções acessórias, face dorsal com células similares, porém menores e arranjadas em 2-3 fileiras.

Material selecionado: Canaã dos Carajás, Serra Sul, S11B, 6²1'21''S, 50²3'27''W, 14.II.2017, D.C. Zappi et al. 3508 (MG); S11C, beira da estrada para lagoa das macrófitas, 02.XII.2015, A.S. Reis et al. 39
(MG); Serra da Bocaina, 6¹8'51' S, 4954'36"W, 712 m, 14.IV.2016, B.F. Falcão et al. 364 (MG); Serra do Tarzan, 6²0'15"S, 5009'55"W, 736 m, 21.VI.2016, B.F. Falcão et al. 536 (MG). Parauapebas, Serra Norte, N4, 606'07'S, 50¹1'14"W, 719 m, 26.III.2016, R.M. Harley et al. 57495 (MG); N6, 607'48''S, 50¹0'36”'W, 693 m, 26.III.2016, B.F. Falcão et al. 245 (MG); N7, 04.II.1985, O.C. Nascimento \& R.P. Bahia 1159 (MG); N8, 609'46"S, 5009'50"W, 695 m, 27.III. 2015, $A$. Cardoso et al. 1952 (MG).

Material adicional selecionado: BRASIL. PARÁ: Curionópilis, Serra do Cristal, canga, 6²7'12'"S, 4940’32”W, 760 m, 04.V.2016, P.L. Viana et al. 6123 (MG). São Felix do Xingu, Serra de Campos, Platô SF, canga, 6³2'34"'S 51 52 '41'”, 660 m, 04.V.2016, P.L.

Viana et al. 6131 (MG).

Espécies e espécimes utilizados para comparação: Portulaca brevifolia Urban, Cuba, C. Wright 3500 (K), tipo da espécie.

Portulaca conicooperculata Poellnitz $=P$. sedoides N.E.Br (Legrand 1962). Brasil, Roraima, Serra do Mel, agosto 1909, fl. branca, E. Ule 8118, tipo de P. conicooperculata (K, MG). Em MG o espécime está identificado como $P$. pilosa, mas deve ser redeterminado como $P$. sedoides.

Portulaca pilosa L. Brasil, Amazonas, São Gabriel da Cachoeira no rio Negro, fl. red, Spruce 2256, dez. 1852 (K, MG); Rio Negro entre Barcelos e São Gabriel, dez 1851, Spruce 1970 (MG); AM?, Santa Isabel, rocha granítica, dezembro 1857, fl. pink, Spruce 1970 (K), mesmo número que o anterior com localidade diferente; Pará, Santarém, 1851, fl. red, Spruce 912 (K).

Portulaca sedifolia N.E.Br: Guyana, Mount Roraima, M.J.J. Quelch \& F. McConell 237 (K), tipo da espécie. Guyana, Rupunouni, C.Appun $1568(\mathrm{~K})$. Guyana, Rupunouni Dist., 2॰56'S 59॰40’W, P.J.M. Mays \& L.L.Y. Wester 4094 (K).

Os espécimes de Carajás foram inicialmente identificados como Portulaca halimoides L. usando Coelho \& Giulietti (2010), pelas flores brancas, apesar de terem os pixídios sésseis. Subsequentemente foram enquadrados em $P$. sect. Catoclasis (Legrand 1962). Trata-se da seção com maior número de espécies nas Américas, e chegamos aos seguintes nomes: $P$. pilosa L., $P$. halimoides L., P. brevifolia Urban e $P$. sedifolia N.E.Br., caracterizadas por serem plantas pequenas, com densidade de tricomas axilares variável e folhas lineares a oblongo-elípticas. Legrand (1962) incluiu Portulaca brevifolia (com distribuição nas Antilhas) e P. halimoides (com distribuição neotropical) como os únicos táxons de $P$. subsect. Pedicellatae Legr., devido a presença de pixídio com $1,5-2,5(-3) \mathrm{mm}$ 

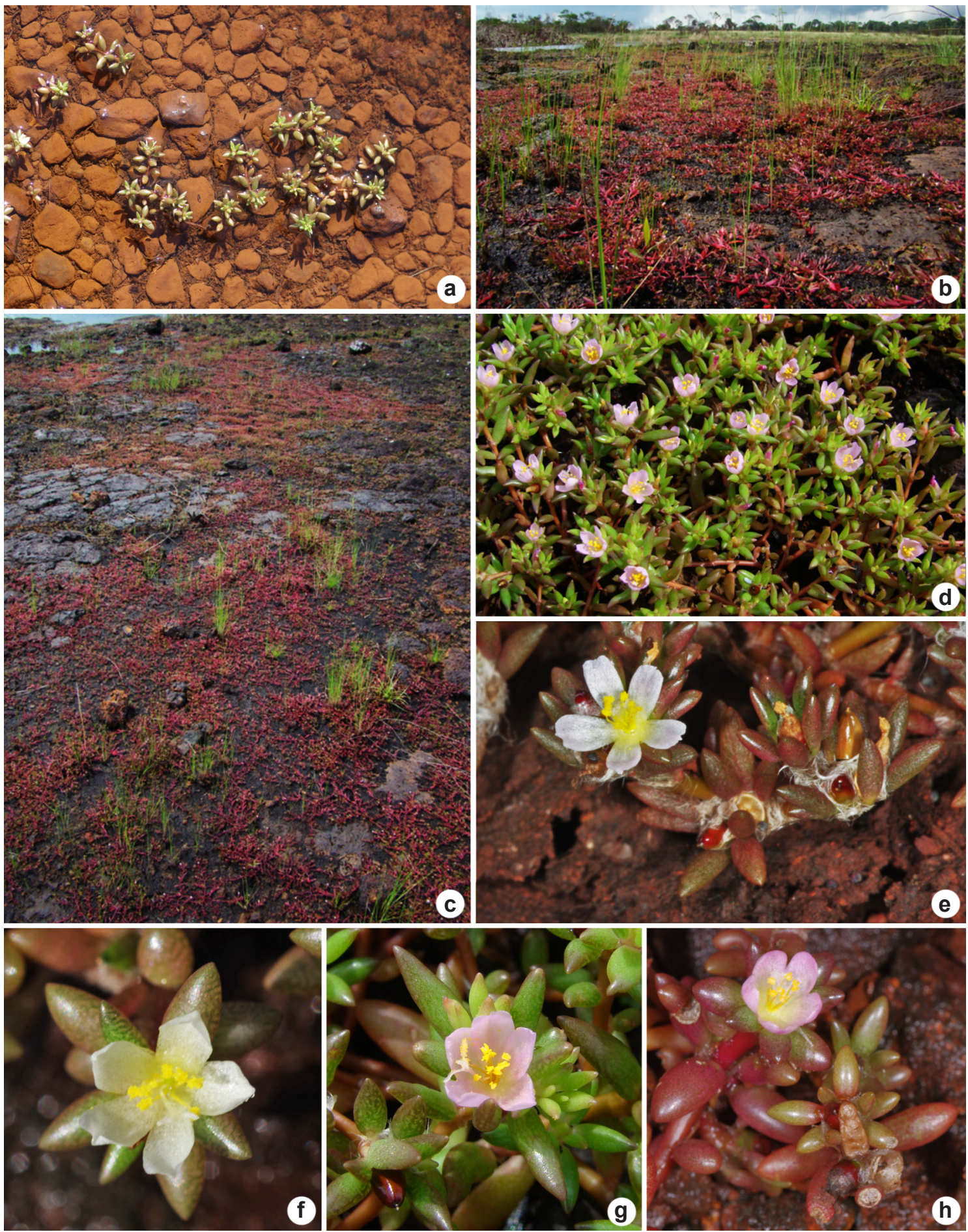

Figura 2 - a-h. Portulaca sedifolia - a. hábitat em período chuvoso; b-c. habitat em período seco, folhas e ramos com coloração avermelhada; d-h. ramos floridos. (Fotos: a, f. Pedro L. Viana; b-d, g. Nara F.O. Mota; e, h. Climbiê Hall). Figure 2 - a-h. Portulaca sedifolia - a. habitat during the rainy season; b-c. habitat during the dry seasons showing reddish leaves and stems; d-h. flowering branches. (Photos: a, f. Pedro L. Viana; b-d, g. Nara F.O. Mota; e, h. Climbiê Hall). 
de comprimento e pedicelos com 1-2,2 mm de comprimento. Steyermark (2005) refere 11 espécies para a Guiana Venezuelana inclusive $P$. pilosa, $P$. halimoides e $P$. sedifolia, distintas especialmente pela cor das flores e tamanho do pedicelo do pixídio. As plantas de Carajás apresentam flores sésseis, brancas a rosadas e pixídio séssil (raramente pedicelo até $0,2 \mathrm{~mm}$ ). Com esse conjunto de caracteres distingue-se de $P$. brevifolia e $P$. halimoides pela ausência de pedicelo no pixídio e de P. pilosa por essa apresentar flores vermelhas ou pink e tricomas axilares densos e longos. Porém, concorda com as características de $P$. sedoides. A confirmação da identificação de Portulaca sedoides para os espécimes de Carajás foi feita com a análise dos materiais-tipo e de outros espécimes complementares em K e MG.

Em herbário, alguns espécimes de $P$. sedifolia com tricomas axilares mais desenvolvidos, podem ser confundidos com espécimes de $P$. pilosa, pois a cor vermelha é perdida na herborização. Porém, as espécies podem ser distintas pela escultura da semente. Em P. sedifolia as sementes medem de 0,6-0,7 $\mathrm{mm}$ compr., as faces laterais apresentam células individuais alongadas a isodiamétricas, paredes anticlinais irregularmente curvadas a retas, paredes periclinais convexas com diminutas projeções acessórias, enquanto a face dorsal apresenta células com formatos similares, porém menores e arranjadas em 2-3 fileiras. Portulaca pilosa segundo Ocampo (2013) têm as sementes com $0,56-0,61 \mathrm{~mm}$, com as células individuais alongadas com paredes anticlinais onduladas com padrões do tipos T-, U-, e V (estreladas) e paredes periclinais convexas e com domo.

O material-tipo de $P$. conicooperculata Poellnitz (E. Ule 8118) tem as sementes com as mesmas características encontradas em $P$. sedifolia e concordamos com a sinonimização proposta por Legrand (1962) apesar do autor referir que não viu os materiais-tipo das duas espécies.

Portulaca sedifolia tem distribuição no Escudo Guiano tendo sido registrada para Venezuela, Guiana, Suriname e Guiana Francesa (Steyermark 2005; Funk et al. 2007). Segundo Steyermark (2005) a espécie ocorre também em áreas do Brasil adjacentes as Guianas e na Colômbia. Nesse trabalho confirmamos a espécie para o estado de Roraima e fazemos a primeira referência para o estado do Pará, onde ocorre na Flona de Carajás (Municípios de Canaã dos Carajás e Paraupebas), no Parque Nacional dos Campos Ferruginosos (Município de Canaã dos Carajás - Serra da Bocaina e Serra do Tarzan) e em outras áreas de cangas da Serra dos Carajás nos Municípios de Curionópilis e São Felix do Xingu.

Na Flona de Carajás ocorre: Serra Norte, N4, N6, N7, N8, na Serra Sul e S11D. Nessas áreas, $P$. sedifolia forma populações com grande número de indivíduos, que ficam presos diretamente sobre as cangas, geralmente em áreas inundáveis durante o período chuvoso. São facilmente reconhecíveis em campo pelo hábito prostrado, folhas pequenas, lineares a elípticolanceoladas, carnosas e pequenas flores brancas a rosadas que abrem uma de cada vez, entre 10-11 horas da manhã (Fig. 2). As plantas completam seu ciclo de vida em 4-5 meses, período coincidente com o das chuvas. No conjunto das populações, a espécie apresenta grande variação morfológica incluindo desde plantas muito pequenas com 3,5-4 cm de comprimento, e pouco ramificadas (Silva 1916) até plantas muito ramificadas, com ramos de até $22 \mathrm{~cm}$ de comprimento (Falcão 443), plantas glabrescentes ou muito pilosas (Falcão 442, Zappi 3508). As plantas muito pilosas, quando herborizadas, são difíceis de distinguir de $P$. pilosa se não haver anotação da cor das pétalas na etiqueta e se não for possível analisar as sementes.

\section{Agradecimentos}

Agradecemos aos Instituto Tecnológico Vale e ao Museu Paraense Emilio Goeldi, a estrutura e o apoio fundamentais ao desenvolvimento do projeto. Aos diversos setores da Vale, o apoio e infra-estrutura de campo. A João Silveira, a confecção da prancha. A Nara Mota, a elaboração da pranchas de fotos das plantas. Ao Pedro Viana, Nara Mota e Climbiê Hall, as fotografias que ilustram esse trabalho, e Pedro Viana, Nara Mota e Daniela Zappi, o acompanhamento em diversas etapas do trabalho. Aos assessores anônimos, as leituras e sugestões apresentadas. Aos curadores dos herbários BHCB, IAN e MG e ao Royal Botanic Gardens de Kew, o exame dos espécimes ali depositados. Ao projeto objeto do convênio MPEG/ITV/FADESP (01205.000250/2014-10) e ao projeto aprovado pelo CNPq (processo 455505/2014-4), o financiamento e suporte dado para realização deste trabalho. Ao CNPq, a bolsa Sênior a A.M. Giulietti. 


\section{Referências}

APGIV - Angiosperm Phylogeny Group (2016) An update of the Angiosperm Phylogeny Group classification for the orders and families of flowering plants: APG IV. Botanical Journal of the Linnean Society 180: $1-20$.

BFG - The Brazil Flora Group (2015) Growing knowledge: an overview of seed plant diversity in Brazil. Rodriguésia 66: 1085-1113. DOI: 10.1590/21757860201566411.

Carolin RC (1993) Portulacaceae. In: Kubitzki K, Rohwer JG \& Bittrich V (eds.) The families and genera of flowering plants. Springer-Verlag, Berlin. Pp. 544555.

Coelho AAOP (2006) Revisão taxonômica das Portulaca L. (Portulacaceae) do Brasil. Tese de Doutorado. Programa de Pós-Graduação em Botânica. Universidade Estadual de Feira de Santana, Bahia. 240p.

Coelho AAOP \& Giulietti AM (2010) O gênero Portulaca (Portulacaceae) no Brasil. Acta Botanica Brasilica 24: 655-670.

Funk V, Hollowell T, Berry O, KelloffC \& Alexander SN (2007) Checklist of the Guiana Shield (Venezuela: Amazon, Bolivar, Delta Amacuro, Guyana, Surinan, French Guiana). Contributions from the United States National Herbarium 55: 1-584.

Geesink R (1969) An account of the genus Portulaca in Indo-Australia and the Pacific (Portulacaceae). Blumea 17: 275-307.

Hernández-Ledesme P, Berendsohn WG, Borsch T, Von Mering S, Akhani H, Arias S, Castañeda-Noa I, Eggli U, Eriksson R, Glores-Olvera H, Fuentes-Bazán S, Kadereit G, Klat C, Korotkova N, Nyffeler R, Ocampo G, Ochoterena H, Oxelman B, Rabeler
RK, Sanchez A, Schlumpberger BO \& Uotila $P$ (2015) A taxonomic backbone for the global synthesis of species diversity in the angiosperm order Caryophyllales. Willdenowia 45: 281-383. DOI:10.3372/wi.45.4530.

Legrand D (1958) Desmembracion del genero Portulaca. Comunicaciones Botânicas del Museo de Historia Natural de Montevideo 34: 1-17.

Legrand D (1962) Las especies americanas de Portulaca. Anales del Museo de Historia Natural de Montevideo 7: 9-147.

Linnaeus C (1753) Species Plantarum. Laurentius Salvius, Stokholm. 560p.

Lorenzi H (2000) Plantas daninhas do Brasil. $3^{\mathrm{a}}$ ed. Editora Plantarum, Nova Odessa. 608p.

Nyffelen R \& Eggli U (2110) Desintegrating Portulacaceae: a new familiar classification of the suborder Portulacineae (Caryophyllales) based on molecular and morphological data. Taxon 59: 227-240.

Ocampo G (2013) Morphological characterization of seeds in Portulacaceae. Phytotaxa 141: 1-24. DOI10.11646/ phytotaxa.141.1.1.

Ocampo G \& Columbus JT (2012) Molecular phylogeny, historical biogeography, and chromosome evolution of Portulaca (Portulacaceae). Molecular Phylogeny Evolution 63: 97-112.

Steyermark J (2005) Portulacaceae. In: Steyermark J, Berry PE, Jatskevych K, Holst BK (eds). Flora of the Venezuelan Guyana. Missouri Botanical Garden Press, St. Louis. Pp. 376-383.

Uotila P, Sennikov AN \& Danin A(2012) The nomenclature of Portulaca oleracea and P. sativa (Portulacaceae). Willdenowia 42: 25-28.

Willis JC (1966) A dictionary of flowering plants and ferns. $7^{\text {a }}$ ed. University Press, Cambridge. 1213p.

\section{Lista de exsicatas}

Arruda AJ 1418 (1.2). Cardoso A 1952 (1.2). Falcão BF 245 (1.2), 302 (1.2), 363 (1.2), 364 (1.2), 442 (1.2), 443 (1.2), 520 (1.2), 536 (1.2), 633 (1.2). Harley RM 57376 (1.2), 57495 (1.2). Nascimento OC 1159 (1.2). Viana P 6131 (1.2), 6177 (1.2), 6194 (1.2), 6223 (1.2). Reis AS 39 (1.2). Silva ASL 1919 (1.2). Zappi DC 3508 (1.2), 3521 (1.1). 
